\title{
Designing a novel pre-hospital treatment decision model based on transcranial ultrasound and clinical assessment for patients with suspected acute stroke: exploratory study
}

Daria Antipova ( $\nabla$ daria.antipova@abdn.ac.uk)

University of Aberdeen Institute of Applied Health Sciences https://orcid.org/0000-0002-0267-1903

Leila Eadie

University of Aberdeen

Helen Shannon

NHS Highland

Philip Wilson

University of Aberdeen

Ashish Macaden

NHS Highland

\section{Research article}

Keywords: stroke; large vessel occlusion; TCD; transcranial ultrasonography; thrombectomy

Posted Date: July 23rd, 2019

DOI: https://doi.org/10.21203/rs.2.11831/v1

License: (c) (i) This work is licensed under a Creative Commons Attribution 4.0 International License.

Read Full License 


\section{Abstract}

Background and objectives Earlier initiation of reperfusion therapy for patients with acute ischaemic stroke is associated with better outcomes. The current exploratory study presents a planned interim analysis aiming to validate a theoretical treatment decision model to select subjects who could benefit from early thrombolysis and/or direct transfer for mechanical thrombectomy. Methods Consecutive subjects suspected of having suffered a stroke within the last 72 hours were recruited. Transcranial greyscale and Doppler ultrasonography was performed within 24 hours of brain computed tomography. The National Institutes of Health Stroke score (NIHSS), modified Rankin Score and medical history were collected retrospectively. Hospital discharge diagnosis was used as the gold standard. Comparative analyses were performed to identify variables that could assist in designing the treatment decision model. Sensitivity, specificity, positive (PPV) and negative predictive values (NPV) were calculated. Results Among a total number of 50 recruited patients, 27 had a final diagnosis of ischaemic stroke, including 10 cases with large vessel occlusion (LVO). Stroke patients were more likely to be older $(p=0.03)$ and to have NIHSS total score $\geq 9(p=0.021)$. NIHSS $\geq 4(p=0.032)$, atrial fibrillation $(p=0.033)$ and cortical signs $(p=0.005)$ were significant indicators of LVO. The proposed model based on grey-scale/Doppler findings and clinical assessment showed $77 \%$ sensitivity and $92 \%$ specificity (77\% PPV, $92 \%$ NPV) for making a decision to initiate reperfusion therapy. Ultrasound findings improved the performance of a model based on clinical data alone (50\% sensitivity, $89 \%$ specificity, $64 \%$ PPV, $82 \%$ NPV). Subjects with a stroke mimicking condition were more likely to have been given reperfusion therapy inappropriately using this model, but no subjects with haemorrhage would have received intravenous thrombolysis. Insufficient acoustic window was seen in approximately $40 \%$ of included participants. Conclusions The proposed treatment decision model might potentially be used for selecting subjects with ischaemic stroke who would benefit from early initiation of reperfusion therapy or immediate transfer for endovascular intervention. A larger sample is required to establish performance of the model with an acceptable degree of precision.

\section{Background}

Standard treatment for acute ischaemic stroke patients include intravenous (IV) administration of a thrombolytic drug. However severe strokes with a mortality rate reaching $80 \%$ [1] are often caused by a large vessel occlusion (LVO), and can be optimally managed by mechanical thrombectomy preceded by IV thrombolysis [2]. While thrombolysis is offered in many regional hospitals, mechanical thrombectomy can only be performed in specialised endovascular centres with neurointerventional facilities.

Administration of each treatment is highly time sensitive. Outcomes are better with shorter delays: the probability of a good clinical outcome decreases by about $11 \%$ with every 30 minute delay from onset to successful reperfusion [3]. However, only $2-10 \%$ of stroke patients worldwide receive IV thrombolysis [4,5] due to various reasons, including late arrival to the hospital, variability in the patient selection criteria, etc [6]. As demonstrated by Ebinger et al (2014), thrombolysis given in the ambulance significantly improved treatment rates with time reduction by at least 15 minutes [7]. 
For patients with suspected LVO, immediate thrombolytic therapy with direct transfer to an endovascular centre ("drip and ship") can potentially lead to more positive outcomes [8]. This model is particularly relevant to remote and rural areas with long travel times.

Designing a triage system for patients with acute ischaemic stroke who would be considered for early reperfusion therapy and/or endovascular intervention has attracted much attention in the last decade. The National Institute of Health Stroke Scale (NIHSS) cutoff ${ }^{3} 11$ and Rapid Arterial Occlusion Evaluation Scale (RACE) cutoff ${ }^{3} 5$ demonstrated the best diagnostic performance however their false-negative rates remain higher than $25 \%$ [9]. Thus, at least $20 \%$ of subjects with LVO would be incorrectly transferred to a local hospital with no neurointerventional facilities available, causing unnecessarily poorer outcomes [9]. Importantly, diagnosis of acute intracranial haemorrhage and/or stroke mimicking condition is often an exclusion criterion in these studies, and therefore their findings are unlikely to be capable of translation into the pre-hospital setting [10].

As suggested by Beume et al (2018), cortical symptoms such as aphasia and/or neglect are indicative of LVO with $91 \%$ sensitivity and $70 \%$ specificity when compared to $85 \%$ sensitivity and $53 \%$ specificity of motor symptoms alone [10]. Similarly, the use of clinical assessment scale thresholds, such as NIHSS ${ }^{3} 10$ $[9,11,12]$, the Field Assessment Stroke Triage for Emergency Destination ${ }^{3} 4$ [12-15] and RACE ${ }^{3} 5$ $[12,14,15]$ which were designed to evaluate cortical symptoms as well as motor symptoms, showed higher sensitivity and specificity when compared to other tools (Antipova et al., 2019, manuscript in preparation). Furthermore, telestroke assessments while in transit supported by hospital-based specialists could improve triage of patients who would benefit from reperfusion therapy [16].

It is crucial to exclude intracranial haemorrhage as one of the absolute contraindications for recanalization therapies. However, scoring tools, such as the Siriraj score, have insufficient precision to predict the presence of haemorrhage reliably [17]. Therefore, brain imaging is currently essential to differentiate haemorrhagic from ischaemic events and to exclude stroke mimics such as tumours $[17,18]$ that would not benefit from reperfusion therapy. However, this is not widely incorporated into the prehospital setting, and is usually performed once a patient arrives in hospital, which may result in treatment delay.

Portable transcranial ultrasound has emerged as a potential method for rapidly assessing the intracranial circulation [19]. Transcranial Doppler (TCD) ultrasound findings, such as absent or diminished blood flow signal in a major intracranial artery in the Circle of Willis were shown to be suggestive of LVO [20-22].

To the best of our knowledge, to date no studies have been published concerning the performance of a combination of clinical assessment and brain imaging with transcranial ultrasonography for selecting subjects who would benefit from early initiation of reperfusion therapy and/or direct transfer for mechanical thrombectomy. We hypothesised that a novel theoretical treatment decision model can be designed to serve this purpose.

Research questions were as follows: 
1. What transcranial grey-scale/TCD ultrasound and clinical assessment findings should contribute to a triage system to correctly select subjects with acute ischaemic stroke who could benefit from early intervention, such as IV thrombolysis and/or mechanical thrombectomy?

2. In what proportion of patients could a correct treatment decision potentially be made using the proposed model?

3. Can the diagnostic accuracy of a treatment decision based exclusively on clinical assessment be improved by adding transcranial grey-scale/TCD ultrasound?

4. In what proportion of suspected stroke patients could transcranial grey-scale/TCD ultrasound not be used due to technical limitations, such as insufficient acoustic window?

As part of a larger study to evaluate the diagnostic precision of a treatment decision tool based on clinical data and transcranial grey-scale/TCD ultrasound, we planned an exploratory evaluation of data from the first 50 eligible patients.

\section{Methods}

\section{Subjects}

An exploratory study was designed as a part of a larger project with the aim of determining which subjects would benefit from early IV thrombolysis and/or direct transfer to an endovascular centre on the basis of transcranial grey-scale/TCD ultrasound and clinical assessment findings.

Data collection was performed from 28 July 2017 to 16 November 2018. Patients suspected of having suffered an acute stroke were recruited into the study upon their admission to Raigmore Hospital (Inverness, UK). Research team members identified eligible candidates on the basis of the following criteria:

Inclusion criteria:

- Suspected stroke patients admitted to Raigmore Hospital for whom standard brain imaging was requested

- Patients with suspected subarachnoid haemorrhage for whom standard brain imaging was requested

- Adults (16 years and over)

- Onset of stroke symptoms not more than 72 hours previously

- Informed consent from the participant, or if they were unable to give meaningful consent, proxy consent from a relative or legal representative in compliance with the Adults with Incapacity Act (Scotland, 2000).

Exclusion criterion: 
- Patients for whom no standard brain imaging was requested.

\section{Data collection}

Baseline demographic and clinical information, including electrocardiography findings, past medical history, symptoms at onset, including cortical symptoms such as neglect, dysphasia or aphasia, and eye deviation, the NIHSS and modified Rankin Scale (mRS) scores were collected retrospectively. The NIHSS total score was calculated using standard procedure [23].

\section{Brain imaging}

According to the National Institute for Health and Care Excellence guideline NG128 (May 2019), brain imaging with a non-enhanced CT is recommended for the early assessment of people with suspected acute stroke. CT was performed as per standard hospital protocol upon patient presentation using a General Electric 750HD G64-slice Discovery or General Electric 64-slice light speed scanner. Diagnosis of acute ischaemic stroke due to LVO was made on the basis of ASPECTS - Alberta stroke programme early CT score.

Transcranial grey-scale/TCD ultrasound was performed within 24 hours of the CT scan using a SonoSite M-Turbo ${ }^{\circledR}$ Point-of-Care ultrasound machine; Philips Sparq or Philips CX50 ultrasound. Since there is no dedicated probe for transcranial scanning available, a 1-5 MHz low-frequency phased array transducer with small footprint was used to allow penetration through the skull. All transcranial grey-scale/TCD scans were obtained by the same medically trained research team member after completing brief training that would reflect the type and volume of training that prehospital clinicians might receive. The researcher performing transcranial ultrasound scanning was blinded to the $\mathrm{CT}$ findings but presenting complaints and/or examination findings were available in all cases. Index test (transcranial grey-scale/TCD ultrasound) results were not available to the assessors of the reference standard (CT), although clinical information was provided.

Participants were asked to remain in a supine or sitting position throughout the ultrasound scanning. Scanning was done through temporal bone windows on either side of the head using grey-scale mode to visualise brain structures and TCD ultrasound for assessment of blood flow in the major intracranial arteries. The operator followed a standard protocol to explore for:

1. Brain structures in the coronal and transverse planes, aiming to visualise any acute intracranial haemorrhage. Acute intracerebral haemorrhage can be detected on ultrasound images as homogenous hyperechogenic structures which are well distinguished from surrounding tissues. Spontaneous subarachnoid haemorrhage can be detected as hyperechogenic signal in the basal cisterns [24].

2. Midline shift by taking three consecutive measurements of the distance between the ultrasound probe and the centre of the third ventricle measured along a line perpendicular to the walls of the 
third ventricle from the ipsilateral side. Midline shift was calculated using the equation provided elsewhere [25].

3. Visualisation of the cerebral peduncles / brainstem.

4. Visualisation of the blood flow in the major intracranial arteries forming the Circle of Willis bilaterally with TCD sonography - middle cerebral artery (MCA), anterior cerebral artery (ACA) and posterior cerebral artery (PCA). MCA was identified as orthograde blood flow at insonation depth of $40-65 \mathrm{~mm}$ and $30-40 \mathrm{~mm}$ for proximal (M1) and distal (M2) segment, respectively. ACA was detected as retrograde flow at the $60-75 \mathrm{~mm}$ depth of insonation. PCA was visualised as orthograde (P1 segment) or retrograde (P2 segment) at 55-75mm depth [26].

LVO was defined as occlusion of one of the major intracranial arteries forming the Circle of Willis, namely M1, M2 segments of the MCA, ACA and PCA. TCD ultrasound diagnosis of the MCA mainstem occlusion was considered when the flow was absent or minimal, blunted, or damped throughout the MCA with increased flow velocity in the anterior or posterior cerebral arteries in comparison to contralateral side (flow diversion) [20-22,27-29]. ACA and PCA occlusion were defined as the absence of the flow or the presence of minimal, blunted, or dampened flow signals throughout these vessels $[22,30]$.

An insufficient temporal bone acoustic window was defined as the inability to visualise the heart-shaped cerebral peduncles as a hypoechoic structure on grey-scale transcranial ultrasound images and/or the major intracranial arteries.

The patient's final discharge diagnosis was referred to as the "gold" standard.

\section{Statistical analysis}

All patients were divided into four groups for the purposes of the comparative analysis as follows: 1 acute ischaemic stroke patients due to LVO; 2 - acute ischaemic stroke patients with no evidence of LVO; 3 - acute haemorrhagic stroke; 4 - stroke mimicking conditions and TIA. The following transcranial greyscale/TCD ultrasound and clinical assessment findings were incorporated into comparative analysis with the aim to identify difference in the outcome between the four groups: presence of haemorrhagic focus on transcranial grey-scale ultrasonography, midline shift (cm), signs of LVO on TCD ultrasound; plus the following clinical data: age (years), presence of cortical signs, NIHSS total score, mRS total score, history or ECG findings of atrial fibrillation, systolic blood pressure levels $(\mathrm{mmHg})$, current anticoagulant or antiplatelet therapy. Statistical analysis was performed using IBM SPSS Statistics 25 software. Distribution shape of the numerical data was analysed to determine the appropriate statistical test for two groups of independent data. Unpaired t-tests were chosen for normally distributed data and Mann-Whitney $U$ tests were employed to analyse skewed data sets with mean and median summary measures reported, respectively. Fisher's exact test was used for analysis of the two groups of independent categorical data. Statistically significant differences between the groups were documented if the probability value $(p)$ was equal or below 0.05

\section{Designing the treatment decision model}


A tentative treatment decision model was designed on the basis of the following data:

1. Statistically significant $(p \leq 05)$ transcranial grey-scale/TCD ultrasound and clinical assessment findings based on the results of the comparative analysis

2. Evidence of any contraindications for IV thrombolysis [31]

3. Clinically important parameters based on previously published data, such as NIHSS ${ }^{3} 10[9,11,12]$ and presence of cortical symptoms [10] for selecting subjects with LVO.

The aim was to determine whether the proposed pathway could be used to select subjects that:

(1) would potentially benefit from early IV thrombolysis (acute ischaemic stroke group with no signs of LVO);

(2) would benefit from a direct transfer to an endovascular centre for mechanical thrombectomy preceded by early IV thrombolysis (acute ischaemic stroke due to LVO group);

(3) would not benefit from reperfusion therapy (patients with a TIA, intracranial haemorrhage, and stroke mimics).

True positive (TP), true negative (TN), false positive (FP) and false negative (FN) rates were obtained on the basis of the proposed treatment decision model. Sensitivity, specificity, positive (PPV) and negative predictive values (NPV) of the model were calculated.

\section{Results}

A total of 50 participants aged 33-92 years old (mean \pm standard deviation, $70.7 \pm 13$ ) meeting the inclusion criteria were selected consecutively upon their admission to the hospital and recruited into the current study (Figure 1).

Figure 1. Flow-diagram. The recruitment of participants into the current study and final analysis.

Among the included participants, 21 were males and 29 were females.

Characteristics of participants are presented in Additional file 1.

All participants were of white ethnic origin. Ischaemic stroke was diagnosed in 27 cases including ten participants with LVO (LVO in the anterior circulation $n=8, L V O$ in the posterior circulation $n=2$ ). Acute intracranial haemorrhage was diagnosed in 10 cases, including 1 participant with subarachnoid haemorrhage. Eight patients had a final diagnosis of a stroke mimicking condition, such as multiple sclerosis, benign paroxysmal positional vertigo, functional neurological disorder, neuropathic pain, Bell's palsy, migraine, hypertension induced paraesthesia, and angioma. Five people had a final diagnosis of a TIA. NIHSS total score varied from 0 to 28 points (mean \pm standard deviation, $5.72 \pm 7$ ). All included participants underwent $\mathrm{CT}$ brain imaging. 


\section{Comparative analysis}

Statistically significant outcomes of the comparative analysis are presented in Additional file 2.

Patients with a confirmed diagnosis of acute stroke were older (median age 74 vs. 58 years, $p=0.03$ ), had a higher total NIHSS score (median score 4 vs. 2, p=0.032), and were more likely to have cortical signs $(p=0.039)$ compared with subjects with a stroke mimicking condition. NIHSS cut-off ${ }^{39} 9$ was a significant predictor of a stroke diagnosis $(p=0.021)$.

Systolic blood pressure was significantly higher in patients with intracranial haemorrhage (median level 171 vs. $153 \mathrm{mmHg}, p=0.135)$. It was also shown that normal blood flow on TCD ultrasound $(p=0.009)$ (Figure 2 ) and presence of haemorrhagic foci on grey-scale $(p=0.001)$ (Figure 3 ) were significantly associated with the diagnosis of intracranial haemorrhage.

Figure 2. TCD ultrasound findings in a patient with a massive right MCA territory infarct. Absent flow in the middle cerebral artery and reversed flow in the ipsilateral anterior cerebral artery (A) when compared to the vessels on the unaffected side (B).

Figure 3. Brain imaging findings in a patient with acute intracranial haemorrhage. CT brain scan (A) and transcranial grey-scale ultrasonography (B) showing a $12 \mathrm{~mm}$ haemorrhagic lesion in the right basal ganglia.

Patients with LVO were more likely to have a high NIHSS score (median score 14 vs. $2, p<0.001$ ) with optimal cut-off of $4(p=0.032)$. Presence of cortical signs $(p=0.005)$ and atrial fibrillation (from history or $E C G)(p=0.033)$ were also significant predictors of LVO.

\section{Treatment decision pathway}

On the basis of the results of the comparative analysis, indications for IV thrombolysis [31] and previously published data $[9,11,12]$, a tentative treatment decision model was designed (Figure 4).

\section{Figure 4. Treatment decision pathway based on the transcranial ultrasound and clinical assessment}

findings. ICH - intracranial haemorrhage; IV - intravenous; LMWH - low molecular weight heparin; LVO large vessel occlusion; TCD - transcranial Doppler ultrasound.

On the basis of the proposed model, $7 / 10$ (70\%) of the sample's patients with LVO would be identified and correctly selected for direct transfer to endovascular centre. All $(16 / 16,100 \%)$ cases of ischaemic stroke not associated with LVO would have been correctly assigned to early thrombolysis. In 20/23 (87\%) cases of patients who would not benefit from IV thrombolysis or endovascular treatment (intracranial haemorrhage, TIA and stroke mimicking conditions), it was correctly decided not to initiate reperfusion therapy.

One case of LVO in the posterior circulation would incorrectly not have received reperfusion therapy due to a misleading mild neurological deficit. In the remaining two cases of LVO that would not have received 
treatment the decision could not be made due to insufficient temporal bone acoustic window and therefore the inability to exclude intracranial haemorrhage on transcranial grey-scale/TCD ultrasound.

2/10 (20\%) subjects with intracranial haemorrhage had an insufficient temporal bone acoustic window, which still led to a treatment decision of not initiating reperfusion therapy, which would have been the correct decision. In $3 / 8$ (38\%) patients who were diagnosed with a stroke mimicking condition the decision would have been made incorrectly to initiate reperfusion therapy, mainly because of the high NIHSS total score and false signs of absent blood flow in MCA on TCD ultrasound. The treatment decision for each case is presented in Additional file 2.

Overall, an insufficient temporal bone acoustic window was documented in 20/50 (40\%) cases. TP, TN, FP, FN rates of the proposed model are presented in Table 1.

Table 1. Positive and negative rates of the model based on transcranial ultrasound and clinical assessment findings.

Theoretical model based on transcranial ultrasound findings and clinical assessment
Decision to

initiate

reperfusion

therapy

Decision NOT to

initiate therapy
Best treatment decision based on final discharge diagnosis

$\begin{array}{ll}\text { Decision to } & \text { Decision NOT } \\ \text { initiate } & \text { to initiate } \\ \text { reperfusion } & \text { therapy } \\ \text { therapy } & \end{array}$

The proposed model showed $77 \%$ sensitivity and $92 \%$ specificity with $77 \%$ PPV, $92 \%$ NPV.

Performance of the model based only on clinical assessment findings, without transcranial greyscale/TCD ultrasound examination, is presented in Table 2.

Table 2. Positive and negative rates of the model based on clinical assessment findings.

Best treatment decision based on final discharge diagnosis

$\begin{array}{ll}\text { Decision to initiate } & \text { Decision NOT to } \\ \text { reperfusion therapy } & \text { initiate therapy }\end{array}$

Theoretical model based on clinical assessment
Decision to initiate reperfusion therapy

Decision NOT to initiate therapy
7

4

7

32 
As demonstrated in Table 2, performance of the model based on clinical findings alone was poorer than the proposed model with a combination of transcranial grey-scale/TCD ultrasound and clinical assessment. It showed a lower sensitivity of $50 \%$, specificity of $89 \%$, PPV of $64 \%$ and NPV of $82 \%$.

\section{Discussion}

Early recognition and initiation of reperfusion treatment is associated with more favourable outcome [32]. However, the diagnostic accuracy of currently available tools does not allow reliable selection of patients who would benefit from a direct transfer to regional endovascular centres for mechanical thrombectomy, potentially leading to inappropriate and costly transfers [9].

Previous research has demonstrated sensitivity of TCD ultrasound ranging from $68 \%$ to $100 \%$ and specificity $78 \%$ to $99 \%$ for detecting acute vessel occlusion and stenosis [20-22,30,33-35]. It is a simple, non-invasive and affordable diagnostic tool that takes $<15$ minutes for a complete assessment of cerebral vessels $[27,36]$ and can be performed in space-restricted environments, such as ambulances $[16,37]$. As demonstrated previously $[16,38,39]$, transcranial grey-scale/TCD ultrasound images can be transferred from remotely supported ambulances in remote and rural areas for hospital-based expert interpretation, which reduces the requirement for skill acquisition and maintenance on the part of the onscene assessor.

In the current exploratory study, we propose that the designed novel treatment decision tool based on transcranial grey-scale/TCD ultrasound together with clinical assessment findings could potentially improve the selection of patients with acute ischaemic stroke who would benefit from early initiation of reperfusion therapy, though further confirmatory work is required. It was demonstrated that the presence of cortical signs was significantly associated with the diagnosis of a stroke in general, and ischaemic stroke in particular. In agreement with previously published data, higher NIHSS total score [11,12], presence of cortical signs [10] and atrial fibrillation were significant predictors of a diagnosis of LVO. Additional features, such as more advanced age, can point towards a more likely diagnosis of acute stroke rather than a stroke mimicking condition.

Results of the diagnostic accuracy analysis of the proposed model show that a correct treatment decision would have been made in $70 \%$ of LVO cases and $100 \%$ of patients with other types of acute ischaemic stroke that were not caused by LVO. Subjects with a LVO in the posterior circulation are likely to be missed due to the poorer sensitivity of TCD ultrasound and clinical assessment tools in this group $[22,35,40]$.

A haemorrhagic focus was detected on transcranial grey-scale/TCD ultrasound only in $50 \%$ of confirmed cases, mainly in central structures, such as basal ganglia. In agreement with previously reported studies [41], haemorrhages were likely to be missed if located in the cortical areas, posterior fossa, or in other cerebral structures that cannot be reached by ultrasound through the transtemporal window (for example, 
the thalamic lesions in cases 15 and 47, Additional file 1). Clinical assessment can therefore provide additional valuable information, for example, history of anticoagulant therapy, active brain tumour, and high systolic blood pressure [42] that would point towards suspected intracranial haemorrhage even in the absence of haemorrhagic foci on transcranial grey-scale ultrasound. A decision not to initiate reperfusion therapy would have been made based on transcranial grey-scale/TCD ultrasound and clinical assessment findings in all cases of intracranial haemorrhage, including those with insufficient acoustic window.

By making an effort to shorten the time interval from stroke symptom onset to delivery of IV thrombolysis, it is highly important to avoid providing this therapy to patients with conditions other than stroke. As demonstrated by Tsivgoulis et al (2015), about $15 \%$ of all patients treated with IV thrombolysis actually have stroke mimics. An inappropriate administration of reperfusion therapy could potentially lead to serious complications, such as intracranial and extracranial bleeding [43] but the risks are relatively low except when haemorrhage is present. In the present study, the highest rate of incorrect treatment decisions was in the stroke mimic group (38\%). These were the subjects with a functional neurological disorder, neuropathic pain with associated weakness, and Bell's palsy. It should be acknowledged that patients with stroke mimicking conditions might be given inappropriate treatment even in the settings of a tertiary care stroke centre [43]. A correct treatment decision was made in all cases of TIA.

Overall performance of the proposed model based on a combination of transcranial grey-scale/TCD ultrasound and clinical assessment was better when compared with a similar system based on clinical findings alone. Interestingly, in one case a stroke mimicking condition was suspected on the basis of clinical assessment and transcranial grey-scale findings, in contrast with the CT findings which reported a bifrontal subarachnoid haemorrhage. A review of the original CT scan revised the final diagnosis to a probable bifrontal angioma.

Further work is needed to refine the model to decrease false positive rates, particularly for stroke mimicking cases, and false negative rates to ensure LVO in posterior circulation are accurately detected.

As demonstrated in the theoretical modelling study by Holodinsky et al (2018), in remote and rural areas with increased transport times, patients with ischaemic stroke due to LVO might benefit from early IV thrombolysis followed by streamlined transfer to an endovascular centre ("drip and ship") [8]. Furthermore, the minimum 15 minute decrease in time to treatment can be achieved by offering thrombolysis in specialised stroke ambulances without increasing the risk of intracerebral haemorrhage or mortality [7]. The current exploratory work is part of a larger ongoing research study aimed at validating a novel triage system to select subjects who would benefit from early reperfusion therapy and direct transfer to a specialised centre. Adequate sensitivity and specificity in such a triage tool could potentially justify a randomised controlled trial of the "drip and ship" model with remotely supported specialist interpretation of the data and decision making.

\section{Limitations}

Page $11 / 21$ 
The current study had several limitations. Firstly, the data in this exploratory study were collected from a sample size of only 50 participants, ten of which were confirmed as having LVO. Some patients with very mild or resolved symptoms might have been missed out from the present study due to early discharge. Equally, patients with a severe stroke may have been not included in the current study due to early mortality. These could potentially result in the studied cohort being unrepresentative of the wider population.

In most cases, the time interval between symptom onset and TCD ultrasound exceeded 24 hours. This could have potentially resulted in some early dynamic changes of blood flow being missed [44]. Future work is required to recruit a larger sample with suspected acute stroke as soon as possible after symptom onset, ideally at the pre-hospital stage, to validate the findings of the current exploratory work.

A proportion of patients with acute ischaemic stroke due to LVO can present with mild neurological deficit [45]. As demonstrated previously, $9-13 \%$ of patients with NIHSS $\leq 4$ had a LVO detected on angiography $[46,47]$ which can be successfully treated with endovascular therapy [48]. The proposed triaging model has been designed to identify cases with a moderate to severe deficit that would benefit from reperfusion therapy. If based purely on clinical assessment, LVO cases with milder symptoms could be missed. We suggest that positive TCD findings might potentially decrease the rate of false negatives.

Angiography was not used as a reference test for detecting LVO as it is not routinely performed in this study centre at present. Therefore, further assessment with CT or magnetic resonance angiography would be recommended to confirm the diagnosis of a major intracranial vessel occlusion. MRI diffusionweighted imaging would be required to confirm the diagnosis of an AIS and to exclude a stroke mimicking condition in those cases where CT imaging was negative.

Multivariate analysis has not been performed as part of the current interim analysis due to the low sample size, however further data analysis on a larger population would be required using a multivariate model which may allow more accurate predictions. Validation in an external cohort is advisable to test the generalisability of the proposed model.

An important and well recognised technical limitation of transcranial grey-scale/TCD ultrasound is the insufficient temporal acoustic window, which has been shown to occur in around $8 \%$ of the European general population [49] to $29 \%$ in the Oriental general population [50]. It is more commonly seen in elderly subjects, especially in women over 50 years old [49]. In our study, an insufficient window was seen in a slightly higher proportion of participants - about $40 \%$. This may be explained by the fact that stroke patients who were generally older were recruited. However, an insufficient window only precluded a treatment decision in 4/50 (8\%) patients. Further work to optimise ultrasound probe design and lowfrequency insonation may be justified.

\section{Conclusions}


A novel treatment decision model for patients with suspected acute stroke based on transcranial ultrasound findings and clinical assessment has been proposed and tested in a small group of 50 subjects. It showed a better diagnostic performance when compared to a similar system using clinical assessment alone. A correct treatment decision was made in around $70 \%$ of patients with LVO and all subjects with other types of ischaemic stroke. Further work is needed to refine the proposed model in a larger sample with acute stroke. If further validated, this system could potentially be implemented in the pre-hospital stroke care setting in remote and rural areas.

\section{List Of Abbreviations}

ACA - anterior cerebral artery; AF - atrial fibrillation; CT - computed tomography; $F N$ - false negative value; FP - false positive value; IV - intravenous thrombolysis; LVO - large vessel occlusion; MCA middle cerebral artery; mRS - the modified Rankin Score; NIHSS - the National Institutes of Health Stroke Scale; NPV - negative predictive value; PCA - posterior cerebral artery; PPV - positive predictive value; RACE - the Rapid Arterial oCclusion Evaluation Scale; TCD - transcranial Doppler; TIA - transient ischaemic attack; TN - true negative value; TP - true positive value.

\section{Declarations}

\section{Ethics approval and consent to participate}

This study was granted ethical approval from Scotland A REC 17/SS/0046 and approved by NHS Highland Research Management, ID 1225.

\section{Consent for publication}

Consent for publication was obtained from participants or their relative / legal representative according to the Adults with Incapacity Act (Scotland, 2000).

\section{Availability of data and materials}

The collected and analysed data are available from the corresponding author upon request.

\section{Competing interests}

The authors declare no competing interests.

\section{Funding}

This work is part of a PhD project supported by the University of Aberdeen's Elphinstone Scholarship Programme. LE's work was funded by the European Space Agency SatCare grant.

\section{Authors' contributions}


AM, PW, LE and DA were involved in the conceptualisation and development of the methodology. DA with help from AM performed transcranial ultrasonography and collected clinical findings. All authors were involved in data analysis. HS reviewed CT brain scans. DA performed statistical analysis and drafted the manuscript. All authors read and approved the final manuscript.

\section{Acknowledgements}

The authors would like to thank Dr Angus Macleod, Professor Stephen Leslie and Dr Stephen Makin for their most valuable feedback.

\section{Additional Materials}

Additional file 1.docx "Characteristics of included participants" (table)

Additional file 2.docx "Results of comparative analysis" contains the following:

Table 1. Difference between the two groups of patients with a stroke and stroke mimics.

Table 2. Difference between the two groups based on transcranial ultrasonography findings.

Table 3. Difference between the two groups on the basis of clinical features.

Additional file 3.docx "Treatment decision based on transcranial ultrasound and clinical assessment and accuracy of the proposed model" (table).

\section{References}

1. Hacke W, Schwab S, Horn M, Spranger M, De Georgia M, von Kummer R. "Malignant" middle cerebral artery territory infarction: clinical course and prognostic signs. Arch Neurol. 1996;53:309-15.

2. Goyal M, Menon BK, van Zwam WH, Dippel DWJ, Mitchell PJ, Demchuk AM, et al. Endovascular thrombectomy after large-vessel ischaemic stroke: a meta-analysis of individual patient data from five randomised trials. Lancet. 2016;387:1723-31.

3. Khatri P, Abruzzo T, Yeatts SD, Nichols C, Broderick JP, Tomsick TA. Good clinical outcome after ischemic stroke with successful revascularization is time-dependent. Neurology. 2009;73:1066-72.

4. Reeves MJ, Arora S, Broderick JP, Frankel M, Heinrich JP, Hickenbottom S, et al. Acute stroke care in the US: results from 4 pilot prototypes of the Paul Coverdell National Acute Stroke Registry. Stroke. 2005;36:1232-40.

5. Katzan IL, Hammer MD, Hixson ED, Furlan AJ, Abou-Chebl A, Nadzam DM. Utilization of intravenous tissue plasminogen activator for acute ischemic stroke. Arch Neurol. 2004;61:346-50.

6. Mikulik R, Bunt L, Hrdlicka D, Dusek L, Vaclavik D, Kryza J. Calling 911 in response to stroke: a nationwide study assessing definitive individual behavior. Stroke. 2008;39:1844-9. 
7. Ebinger M, Winter B, Wendt M, Weber JE, Waldschmidt C, Rozanski M, et al. Effect of the use of ambulance-based thrombolysis on time to thrombolysis in acute ischemic stroke: a randomized clinical trial. JAMA. 2014;311:1622-31.

8. Holodinsky JK, Williamson TS, Demchuk AM, Zhao H, Zhu L, Francis MJ, et al. Modeling Stroke Patient Transport for All Patients With Suspected Large-Vessel Occlusion. JAMA Neurol. 2018;75:1477-86.

9. Turc G, Maier B, Naggara O, Seners P, Isabel C, Tisserand M, et al. Clinical Scales Do Not Reliably Identify Acute Ischemic Stroke Patients With Large-Artery Occlusion. Stroke. 2016;47:1466-72.

10. Beume L-A, Hieber M, Kaller CP, Nitschke K, Bardutzky J, Urbach H, et al. Large Vessel Occlusion in Acute Stroke. Stroke. 2018;49:2323-9.

11. Vanacker P, Heldner MR, Amiguet M, Faouzi M, Cras P, Ntaios G, et al. Prediction of Large Vessel Occlusions in Acute Stroke: National Institute of Health Stroke Scale Is Hard to Beat. Crit Care Med. 2016;44:e336-43.

12. Purrucker JC, Härtig F, Richter H, Engelbrecht A, Hartmann J, Auer J, et al. Design and validation of a clinical scale for prehospital stroke recognition, severity grading and prediction of large vessel occlusion: The shortened NIH Stroke Scale for emergency medical services. BMJ Open. 2017;7.

13. Zhao H, Pesavento L, Coote S, Rodrigues E, Salvaris P, Smith K, et al. Ambulance Clinical Triage for Acute Stroke Treatment: Paramedic Triage Algorithm for Large Vessel Occlusion. Stroke. 2018;49:945-51.

14. Lima FO, Silva GS, Furie KL, Frankel MR, Lev MH, Camargo ECS, et al. Field Assessment Stroke Triage for Emergency Destination: A Simple and Accurate Prehospital Scale to Detect Large Vessel Occlusion Strokes. Stroke. 2016;47:1997-2002.

15. Gropen TI, Boehme A, Martin-Schild S, Albright K, Samai A, Pishanidar S, et al. Derivation and Validation of the Emergency Medical Stroke Assessment and Comparison of Large Vessel Occlusion Scales. J Stroke Cerebrovasc Dis. 2018;27:806-15.

16. Eadie L, Regan L, Mort A, Shannon H, Walker J, MacAden A, et al. Telestroke assessment on the move: prehospital streamlining of patient pathways. Stroke. 2015;46:e38-40.

17. Hui ACF, Wu B, Tang ASY, Kay R. Lack of clinical utility of the Siriraj Stroke Score. Intern Med J. 2002;32:311-4.

18. Weir CJ, Murray GD, Adams FG, Muir KW, Grosset DG, Lees KR. Poor accuracy of stroke scoring systems for differential clinical diagnosis of intracranial haemorrhage and infarction. Lancet. 1994;344:999-1002.

19. Martinez-Sanchez P, Serena J, Alexandrov A V, Fuentes B, Fernandez-Dominguez J, Diez-Tejedor E. Update on ultrasound techniques for the diagnosis of cerebral ischemia. Cerebrovasc Dis. 2009;27 Suppl 1:9-18.

20. Bar M, Skoloudik D, Roubec M, Hradilek P, Chmelova J, Czerny D, et al. Transcranial duplex sonography and CT angiography in acute stroke patients. J Neuroimaging. 2010;20:240-5. 
21. Wada K, Kimura K, Minematsu K, Yasaka M, Uchino M, Yamaguchi T. Combined carotid and transcranial color-coded sonography in acute ischemic stroke. Eur J Ultrasound. 2002;15:101-8.

22. Tsivgoulis G, Sharma VK, Lao AY, Malkoff MD, Alexandrov A V. Validation of transcranial Doppler with computed tomography angiography in acute cerebral ischemia. Stroke. 2007;38:1245-9.

23. NIH Stroke Scale Instructions [Internet]. [cited 2019 Jan 22]. Available from: https://www.stroke.nih.gov/resources/scale.htm

24. Olatunji RB, Ogbole GI, Atalabi OM, Adeyinka AO, Lagunju I, Oyinlade A, et al. ROLE OF TRANSCRANIAL COLOUR-CODED DUPLEX SONOGRAPHY IN STROKE MANAGEMENT - REVIEW ARTICLE. West African J ultrasound. 2015;16:33-42.

25. Stolz E, Gerriets T, Fiss I, Babacan SS, Seidel G, Kaps M. Comparison of transcranial color-coded duplex sonography and cranial CT measurements for determining third ventricle midline shift in space-occupying stroke. AJNR Am J Neuroradiol. 1999;20:1567-71.

26. Alexandrov A V, Sloan MA, Wong LKS, Douville C, Razumovsky AY, Koroshetz WJ, et al. Practice standards for transcranial Doppler ultrasound: part l--test performance. J Neuroimaging. 2007;17:11-8.

27. Gerriets T, Goertler M, Stolz E, Postert T, Sliwka U, Schlachetzki F, et al. Feasibility and validity of transcranial duplex sonography in patients with acute stroke. J Neurol Neurosurg Psychiatry. 2002;73:17-20.

28. Seidel G, Cangur H, Albers T, Burgemeister A, Meyer-Wiethe K. Sonographic evaluation of hemorrhagic transformation and arterial recanalization in acute hemispheric ischemic stroke. Stroke. 2009;40:119-23.

29. Kim Y, Sin D-S, Park H-Y, Park M-S, Cho K-H. Relationship between flow diversion on transcranial Doppler sonography and leptomeningeal collateral circulation in patients with middle cerebral artery occlusive disorder. J Neuroimaging. 2009;19:23-6.

30. Tsivgoulis G, Sharma VK, Hoover SL, Lao AY, Ardelt AA, Malkoff MD, et al. Applications and advantages of power motion-mode Doppler in acute posterior circulation cerebral ischemia. Stroke. 2008;39:1197-204.

31. Fugate JE, Rabinstein AA. Absolute and Relative Contraindications to IV rt-PA for Acute Ischemic Stroke. The Neurohospitalist. 2015;5:110-21.

32. Goyal M, Jadhav AP, Bonafe A, Diener H, Pereira VM, Levy E, et al. Analysis of workflow and time to treatment and the effects on outcome in endovascular treatment of acute ischemic stroke: Results from the SWIFT PRIME randomized controlled trial. Radiology. 2016;279:888-97.

33. Brunser AM, Lavados PM, Hoppe A, Lopez J, Valenzuela M, Rivas R. Accuracy of transcranial Doppler compared with CT angiography in diagnosing arterial obstructions in acute ischemic strokes. Stroke. 2009;40:2037-41.

34. Guan J, Zhou Q, Ouyang H, Zhang S, Lu Z. The diagnostic accuracy of TCD for intracranial arterial stenosis/occlusion in patients with acute ischemic stroke: the importance of time interval between detection of TCD and CTA. Neurol Res. 2013;35:930-6. 
35. Rathakrishnan R, Berne YI, Quek KK, Hong CS, Ong BK, Chan BP, et al. Validation of transcranial Doppler with CT angiography in cerebral ischaemia: a preliminary pilot study in Singapore. Ann Acad Med Singapore. 2008;37:402-5.

36. Goertler M, Kross R, Baeumer M, Jost S, Grote R, Weber S, et al. Diagnostic impact and prognostic relevance of early contrast-enhanced transcranial color-coded duplex sonography in acute stroke. Stroke. 1998;29:955-62.

37. Marsh-Feiley G, Eadie L, Wilson P. Telesonography in emergency medicine: A systematic review. PLoS One. 2018;13:e0194840.

38. Mort A, Eadie L, Regan L, Macaden A, Heaney D, Bouamrane M-M, et al. Combining transcranial ultrasound with intelligent communication methods to enhance the remote assessment and management of stroke patients: Framework for a technology demonstrator. Health Informatics J. 2016;22:691-701.

39. Eadie L, Mulhern J, Regan L, Mort A, Shannon H, Macaden A, et al. Remotely supported prehospital ultrasound: A feasibility study of real-time image transmission and expert guidance to aid diagnosis in remote and rural communities. J Telemed Telecare. 2018;24:616-22.

40. Ollikainen JP, Janhunen H V, Tynkkynen JA, Mattila KM, Halinen MM, Oksala NK, et al. The Finnish Prehospital Stroke Scale Detects Thrombectomy and Thrombolysis Candidates-A Propensity ScoreMatched Study. J Stroke Cerebrovasc Dis. 2018;27:771-7.

41. Kukulska-Pawluczuk B, Ksiazkiewicz B, Nowaczewska M. Imaging of spontaneous intracerebral hemorrhages by means of transcranial color-coded sonography. Eur J Radiol. 2012;81:1253-8.

42. Jin H-Q, Wang J-C, Sun Y-A, Lyu P, Cui W, Liu Y-Y, et al. Prehospital identification of stroke subtypes in Chinese rural areas. Chin Med J (Engl). 2016;129:1041-6.

43. Tsivgoulis G, Zand R, Katsanos AH, Goyal N, Uchino K, Chang J, et al. Safety of intravenous thrombolysis in stroke mimics: prospective 5-year study and comprehensive meta-analysis. Stroke. 2015;46:1281-7.

44. Kenton AR, Martin PJ, Abbott RJ, Moody AR. Comparison of transcranial color-coded sonography and magnetic resonance angiography in acute stroke. Stroke. 1997;28:1601-6.

45. Heldner MR, Zubler C, Mattle HP, Schroth G, Weck A, Mono M-L, et al. National Institutes of Health stroke scale score and vessel occlusion in 2152 patients with acute ischemic stroke. Stroke. 2013;44:1153-7.

46. Heldner MR, Hsieh K, Broeg-Morvay A, Mordasini P, Bühlmann M, Jung S, et al. Clinical prediction of large vessel occlusion in anterior circulation stroke: mission impossible? J Neurol. 2016;263:163340.

47. Moore RD, Jackson JC, Venkatesh SL, Quarfordt SD, Baxter BW. Revisiting the NIH Stroke Scale as a screening tool for proximal vessel occlusion: Can advanced imaging be targeted in acute stroke? J Neurointerv Surg. 2016;8:1208-10.

48. Kaesmacher J, Chaloulos-lakovidis P, Panos L, Mordasini P, Heldner MR, Kurmann CC, et al. Clinical effect of successful reperfusion in patients presenting with NIHSS < 8: data from the BEYOND-SWIFT 
registry. J Neurol. 2019;266:598-608.

49. Marinoni M, Ginanneschi A, Forleo P, Amaducci L. Technical limits in transcranial Doppler recording: inadequate acoustic windows. Ultrasound Med Biol. 1997;23:1275-7.

50. Hashimoto H, Etani H, Naka M, Kinoshita N, Nukada T. [Assessment of the rate of successful transcranial Doppler recording through the temporal windows in Japanese with special reference to aging and sex]. Nihon Ronen Igakkai Zasshi. 1992;29:119-22.

\section{Figures}

\section{Enrolment}

\section{Assessment for eligibility}

Analysis

\section{Included into the study}

$(n=50)$

Exclusion on the following basis:

- not meeting inclusion criteria:

- no CT brain scan requested;

- time from symptom onset $>72 \mathrm{~h}$;

- time from CT brain scan $>24 \mathrm{~h}$;

- declined to participate

Figure 1

Flow-diagram. The recruitment of participants into the current study and final analysis.

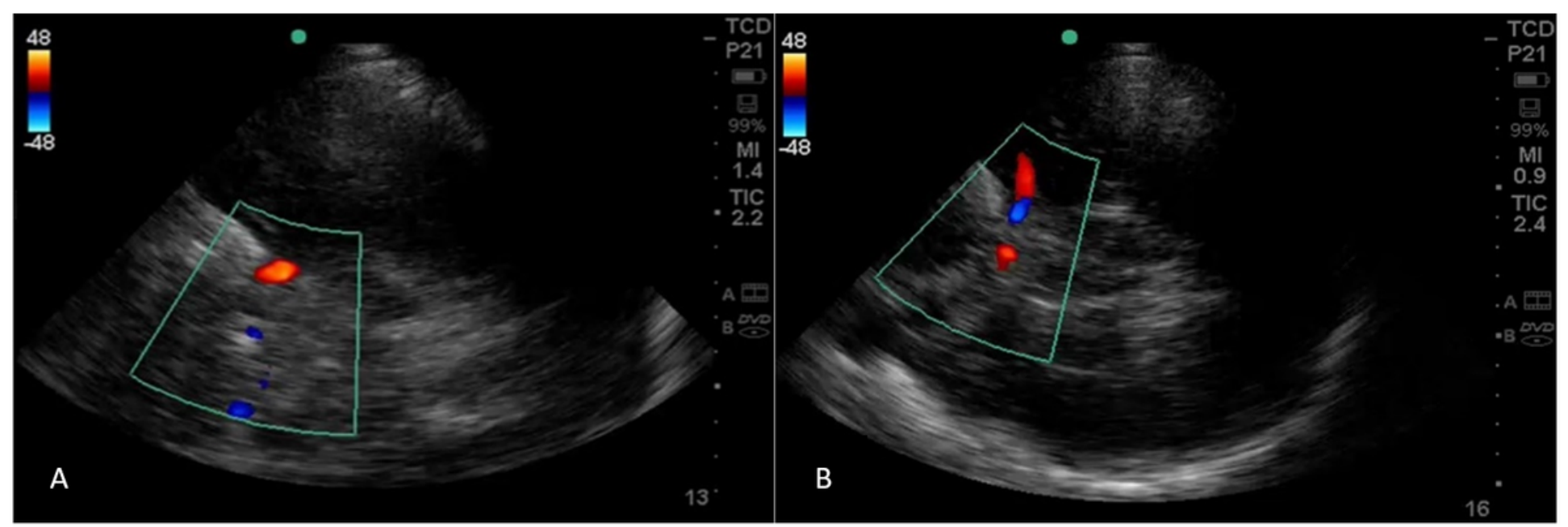




\section{Figure 2}

TCD ultrasound findings in a patient with a massive right MCA territory infarct. Absent flow in the middle cerebral artery and reversed flow in the ipsilateral anterior cerebral artery $(A)$ when compared to the vessels on the unaffected side (B).

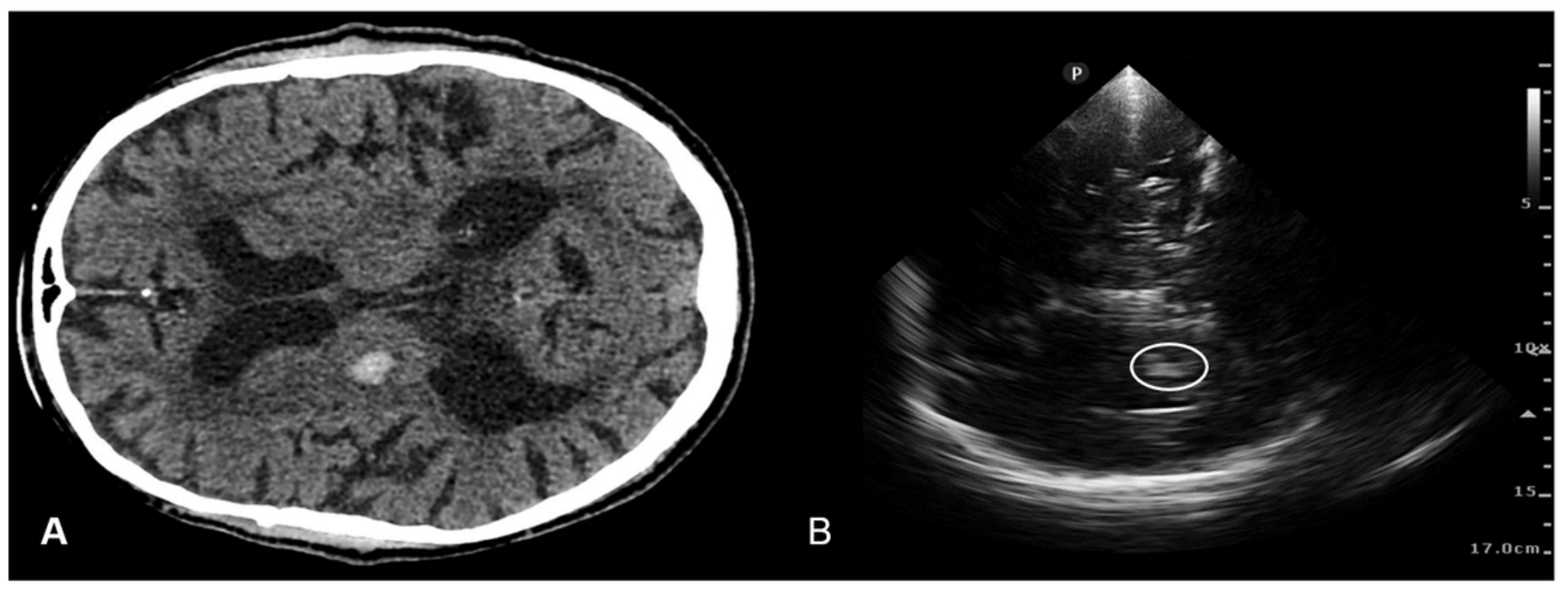

\section{Figure 3}

Brain imaging findings in a patient with acute intracranial haemorrhage. CT brain scan (A) and transcranial grey-scale ultrasonography (B) showing a $12 \mathrm{~mm}$ haemorrhagic lesion in the right basal ganglia. 


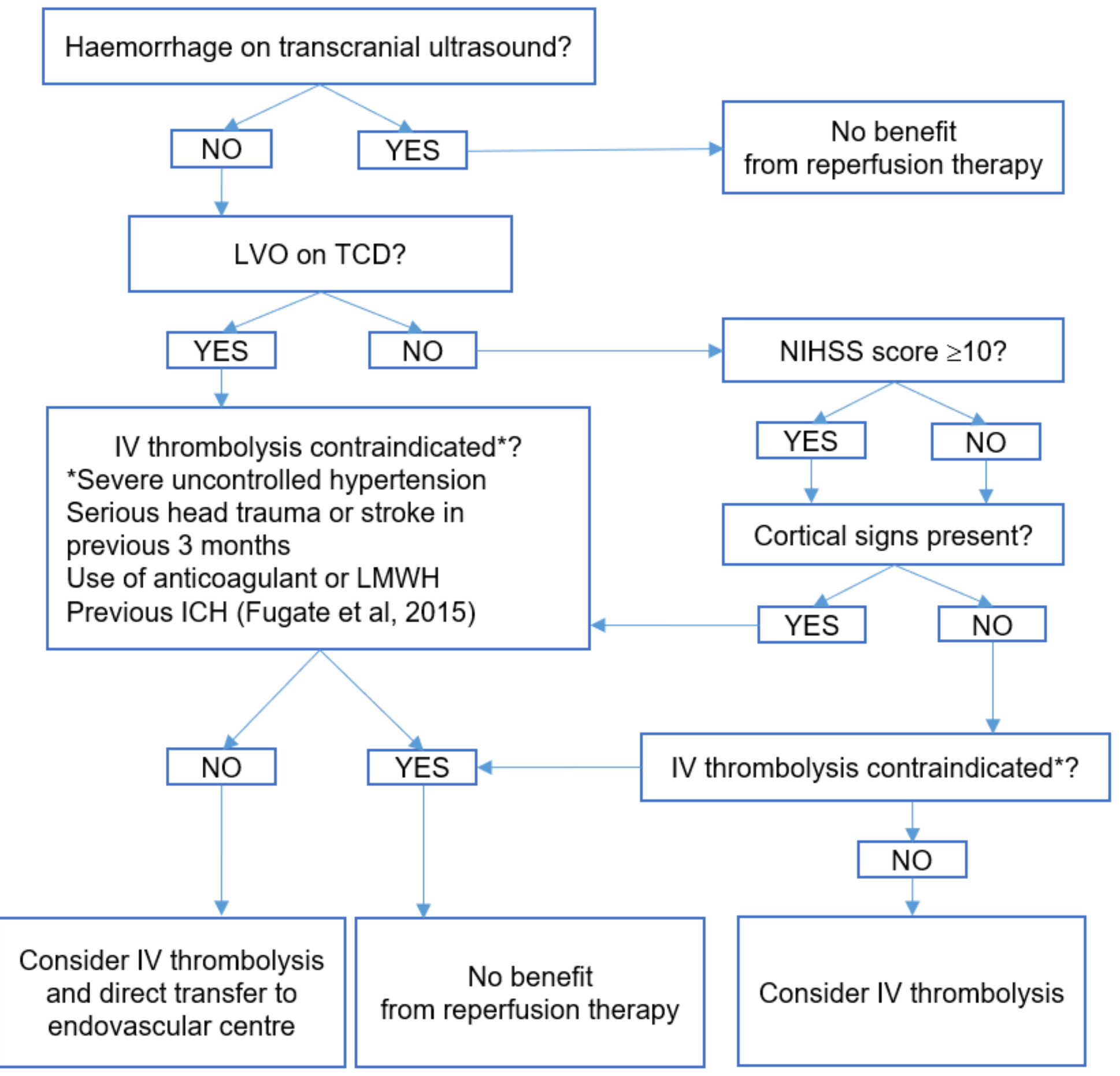

\section{Figure 4}

Treatment decision pathway based on the transcranial ultrasound and clinical assessment findings. ICH - intracranial haemorrhage; IV - intravenous; LMWH - low molecular weight heparin; LVO - large vessel occlusion; TCD - transcranial Doppler ultrasound.

\section{Supplementary Files}


This is a list of supplementary files associated with this preprint. Click to download.

- Additionalfile1.pdf

- Additionalfile3.pdf

- Additionalfile2.pdf 